\title{
THE VARIATIONS IN SCARLET FEVER OF THE OPSONIC POWER OF STREPTOCOCCI. ${ }^{1}$
}

\author{
By A. G. Banks, M.D.(Glasg.), D.P.H.(Lond.), Assistant Medical \\ Officer, Metropolitan Asylums Board.
}

THE general question of immunity in streptococcus infection has been the subject of considerable investigation.

The probable nature and action of the substances present in the blood serum of animals immunised against streptococci was first pointed out by Denys and Leclef (1895 and 1897). These observers immunised rabbits, using cultures of streptococei from various sources and of exalted virulence, and found that the blood then possessed some degree of bactericidal power. No such power (for streptococci) could be demonstrated in either normal or immune sera. This observation has been amply confirmed by Hektoen (1906), Bordet (1895, 1896, 1897), Wright and Windsor (1902), and others. From their experiments they further deduce that the serum acquires something in the process of immunisation which neutralises something in the cocci by reason of which these are protected against phagocytosis.

Marchand (1898), from an elaborate study of phagocytosis of virulent and a virulent streptococci, drew conclusions similar to those of Denys and Leclef. Tchistovitch (1900), on the other hand, from examination of the organs of rabits injected with virulent streptococci, considered that the varying phagocytosis there met with might be due to the fact that not all of the cocci in a culture are of equal virulence.

Denys and Marchand (1896), had found that the addition of 1 per cent. immune horse serum to a suspension of rabbits' leucocytes caused greater phagocytosis than when to the same suspension was added 1 per cent. normal horse serum.

Bordet, discussing Marmorek's anti-streptococcus serum, agrees with the latter that it possesses no bactericidal power, nor does it confer any such power on the animal injected. He attributes to the immune serum a stimulating action on the leucocytes, which manifest a positive chemiotaxis in the case of streptococci. Denys and Mennes (1886-97) pointed out, however, that the essential phenomenon is a

${ }^{1}$ Abstract of a thesis for the degree of Doctor of Medicine in the University of Glasgow.

8 - JL. OF PATH.-YOL. XII. 
phagocytosis of which the primum movens is the antistreptococcus serum. Mennes (1897) obtained results with pneumococci similar to those of Denys and Leclef with streptococci : no bactericidal power had been acquired, but the serum had itself been so changed as to facilitate phagocytosis. Sir A. E. Wright's discovery of specific opsonins, first described by him in the case of staphylococci and tubercle bacilli, and subsequently for many other organisms, including streptococci, confirmed and extended the results of these earlier observers.

Dealing more particularly with streptococci, Neufeld and Rimpau (1904) made the discovery independently, their conclusions agreeing generally with those of Wright. Their experiments with antistreptococcus serum seem to negative Bordet's idea of its action; they show that it exerts an opsonic and not a stimulating effect. Further, in certain diseases caused by organisms allied in respect of the production of anti-bodies in corpore to the streptococcus, e.g. tuberculosis and pneumonia, variations in the progress of the disease have been shown to coincide with alterations in the opsonic index of the individual's serum. Having in view the part played by the streptococcus, especially in the complications of scarlet fever, I have endeavoured to find if any such relation could be demonstrated here. Apart from some more or less indirect observations of Hektoen (1906) and of Ruediger's (1905), I am not aware of any published work on the subject.

The method adopted was that of Wright,-now sufficiently well known, - certain modifications in detail being made to suit the organism used.

One of the chief difficulties was to get a uniform emulsion, as the tendency of some strains to agglutinate is great, and this quality is more marked the older the strain. It was found that growing an easily agglutinated strain in broth for a few days and subculturing on agar lessened this property very decidedly. Heating to $60^{\circ} \mathrm{C}$. seems also to help, but is apt to interfere with subsequent staining.

To stain I have generally used either J. Homer Wright's or Leishman's mixture; but simple stains, such as Loeffler's blue or carbol-thionin blue, are equally satisfactory.

In counting, all clumps are neglected, and each coccus counts as one, degenerated (i.e. unstained) organisms not being included. The question of the exact relative value of a single coccus and a chain is debatable, but the above procedure seems to be the more accurate and gives uniform results.

Four strains of streptococci were used, two of which presented many of the characteristics of streptococcus conglomeratus (Gordon, 1898-1901, Klein, 1886-87) ; the third was probably Streptococcus pyogenes; while the last occupied an intermediate position so far as tested, but on the whole was more closely allied to the first two.

The more important cultural and morphological characters are as under :-

(a) Isolated in practically pure culture from the throat of a mild convalescent case of scarlet fever. In broth, chain formation well developed and marked agglomeration of masses at bottom of medium which remained clear. On agar many bacillus-like and elongated forms were present. Litmus milk 
was firmly coagulated in thirty-six hours, with abundant acid formation. After twelve days or so, slight liquefaction of the agar medium was noted, and the growth thereon was slow. Subcultures on serum showed gradual loss of staining properties, which were, however, recovered after some days' growth in nutrient bouillon. (This phenomenon was noted in two days' old cultures, or even sooner, and did not appear to be due to disintegration of the organisms.)

(b) Obtained in pure culture from a case of tonsillitis during convalescence from scarlet fever. Bacillus and spindle forms on agar were numerous, on serum less abundant, but on the latter medium pleomorphism was very conspicuous. In broth the chains were short, and agglomeration intense. Milk was slowly curdled, with moderate acid formation.

(c) Got in pure culture from the cerebro-spinal fluid of a child with meningitis following mastoid disease. It formed long chains in broth, but no definite masses, the medium also remaining clear. On agar and serum three days' old cultures showed considerable pleomorphism, but no definite spindles nor bacillary forms. Milk was not curdled, and acid production was slight.

(d) Isolated from a cervical abscess occurring in the third week of disease. It presented the typical conglomerate appearance in broth, though not very intensely, was extremely pleomorphic in agar condensation fluid and on serum, but only a few elongated elements could be found, and then after prolonged culture. After two days, slight acid formation and curdling of milk were noted.

As it was not feasible to isolate a special streptococcus from each case observed, these known strains were used throughout the estimations.

In most of the serial observations my own blood was taken as the control, but in others that of nurses in the hospital was used. I have therefore determined, as against my own taken as unity, the indices of these control sera, and to the list is added that of a boy convalescent from a mild attack of varicella. The average for the six was 1.03 , distributed thus :-A. S., 0.99 ; A. B., 1.01 ; A. J. (varicella case), 0.8 ; L. S., 1.03 ; W. B., 1.19 ; and M. C., 1.2 : in the last case a later examination gave an index of 1:01. The three lowest indices are those of individuals who denied having had scarlet fever, but the number is too small and the chance of error in the history too great to admit of any definite inference. As in the case of tubercle bacilli, staphylococci, etc., the normal index for streptococci would seem to vary in healthy people between 0.8 and 1.2 , though borderland indices in apparently healthy individuals should be carefully scrutinised.

A series of five cases of scarlet fever, running fairly normal courses, were investigated, with special reference to the behaviour of the index during convalescence.

CASE 1.-Moderately severe attack, complicated by secondary cervical adenitis : a culture from the throat at this time showed only streptococci.

Opsonic index on the 5 th day of disease, $1 \cdot 4$

\begin{tabular}{|c|c|c|c|c|}
\hline , &  & 11 th & 9 & 0.8 \\
\hline , & , & 19th & ", & 0.63 \\
\hline , & , & 27 th & ,' & 0.87 \\
\hline " & " & 37 th & $"$ & 0.8 \\
\hline " & $"$ & $46 \operatorname{th}$ & $\eta$ & 0.87 \\
\hline
\end{tabular}

CASE 2.-Rather severe initial symptoms, with adenitis and a trace of 
albumin in the urine: the temperature began to fall on the fifth day, and convalescence was uninterrupted.

Opsonic index on the 6 th day of disease, $1 \cdot 2$

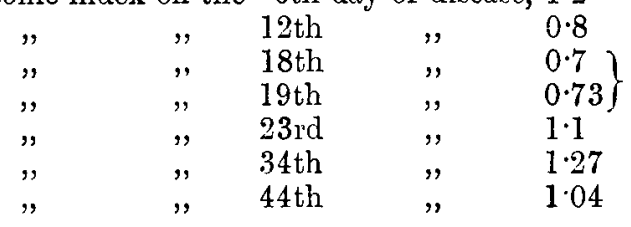

CASE 3.-Of moderate severity, but uncomplicated except for persistent rhinorrhœa, which gave pure cultures of streptococcus.

Opsonic index on the 4 th day of disease, 0.73

$\begin{array}{lllll}" & \text { " } & \text { 7th } & , & 0.94 \\ , & \text { " } & 12 \mathrm{th} & , & 0.6 \\ " & \text { " } & 21 \mathrm{st} & , & 0.72 \\ " & \text { " } & 33 \mathrm{rd} & , & 1.1\end{array}$

On the last occasion the patient's serum was sent from one of the convalescent hospitals, at least sixteen hours having elapsed since withdrawal. The result indicates no marked loss of opsonic power.

CASE 4.-Fairly sharp attack, with trace of albumin in the urine and some rheumatic pains in knees and ankles.

Opsonic index on the 4 th day of disease, 0.82

$\begin{array}{lllll}", & \text { 11th } & , & 0.51 \\ ", & \text { " } & 19 \text { th } & \text { ", } & 0.76 \\ \text {, } & \text { 23rd } & \text { " } & 0.9\end{array}$

Case 5.-Slight attack, the temperature being normal two days after admission.

Opsonic index on the 3rd day of disease, 1.62

$\begin{array}{lllll}\text {, } & \text { " } & 11 \text { th } & , & 1 \cdot 1 \\ " & \text { ", } & 17 \text { th } & , & 0.9 \\ \text { ", } & \text { " } & 30 \text { th } & , & 0.93 \\ \text { ", } & 3 \text { th } & , & 0.97\end{array}$

In the first four cases strains $a$ and $b$ were used, and in Case 5 strain $d$. In all a general correspondence in the opsonic curves is present. In three of them, Cases 1,2, and 5, the initial estimation, made during the fall in temperature, is above normal. In the other two the index during the same period is below normal, the subsequent rapid fall to 0.5 in Case 4 , and 0.6 in Case 3, on the eleventh day being noteworthy. As no marked difference in the length or severity of the fever existed in any, the discrepancy cannot be ascribed to this cause. The type of curve is, however, despite these preliminary differences, much the same in all. During the second week of the disease a marked fall occurs; in most of the cases the index is not above 0.8 , and in Cases 3 and 4 considerably lower. This decline is, generally speaking, continued into the third week, and probably reaches its maximum some time in this week. It is well shown in the first two cases, probably holds true for Case 5, and though in the two remaining cases the secondary rise has apparently set in, the indices during the third week are in both instances below $0 \cdot 8$. 
In this connection an observation made by Hektoen is of interest. He says :

"The lack of clinical evidence of marked active immunity after streptococcus and other infections of this class would seem to indicate that postinfectious opsonic increase is only of brief duration."

This statement is in complete accordance with these results, the opsonic index in the five cases falling in the average five points in nine days, from normal or over to sub-normal. In all five charts the curve tends upwards during the fourth and fifth weeks, the final estimations varying between 0.9 and $1 \cdot 0$. In this series of cases two complications were noted. In Case 4 a trace of albumin was present on admission, and lasted five days. The relatively low indices observed here suggest that these and the albuminuria are to be correlated, though the influences tending to reduce the opsonic power have not been sufficient to disturb the general character of the curve.

In Case 1 secondary or late adenitis supervened on the twentyninth day of disease. An estimation made eight days after, revealed a lower index than before this complication, but subsequently the index rose to $0 \cdot 9$. This illustrates a point which I think may fairly be deduced from these and other considerations to be presently discussed, namely, that a low opsonic index to the streptococcus indicates a liability to further infection of the same type, i.e. streptococcal, but that other attendant and favouring conditions are probably required to enable the organisms to regain a hold. The actual condition of the tonsillar tissue is possibly the chief determining factor, as Tchitchkine (1906) has found, on feeding rabbits with streptococci, that, in the septicæmia induced, infection took place almost exclusively from the first part of the digestive tract, the tonsils being especially liable to invasion.

In three fatal cases observations were made.

CaSE 6.-Very severe, with deep ulceration of tonsils and extensive cellulitic infiltration of neck, from which a pure culture of streptococcus was obtained. There was much sloughing of the cervical tissues, followed by ulceration of the larynx, and death on the twenty-fifth day of disease.

Opsonic index on the 6 th day of disease, 0.74

$\begin{array}{llrll}" & " & \text { 9th } & " & 0.65 \\ " & " & \text { 13th } & " & 1.16 \\ " & " & \text { 17th } & , & 0.7 \\ " & \text { 23rd } & \text { 23rd } & 0.61\end{array}$

CASE 7.-Very severe, with considerable ulceration of tonsils and bilateral cervical adenopathy, albumin and casts in urine on admission, and later complete anuria.

$\begin{array}{ccccc}\text { Opsonic index on the 3rd day of disease, } & 1.0 \\ , " & \text { " } & 11 \text { th } & 0.81 \\ \text { " } & 14 \text { th } & \text { ", } & 0.68\end{array}$

CASE 8.-Severe septic attack, with deep ulceration of tonsils, hæmorrhagic. rash, and cervical swelling. 
The opsonic index on the day preceding death was 0.55 .

In these cases also strains $a$ and $b$ were employed.

Though the number of observations is not great, the range of the index is such as might be expected in cases of septicæmia. That uncharted fluctuations occurred is likely, but the index probably does not in such (fatal) cases rise much above normal. The rise noted in Case 6 certainly coincided with some improvement in the general condition, but so also did the succeeding fall. The only inference I would draw is that the general tendency is for the opsonic power to decrease in cases likely to prove fatal, and that shortly before death the index is low. Wright has shown that in generalised infections the opsonic power varies markedly from time to time, and, contrary to the findings in these cases, may be considerably higher than normal immediately prior to death. G. G. Macdonald (1905) and Rosenow (1906), in the case of pneumonia, have obtained results more analogous to mine. The former finds that at the commencement of the infection, while the temperature is still rising and during the fastigium, the opsonic content is below that of a normal individual. With the onset of the crisis there is a striking rise in the opsonin, which passes normal limits and may give an index of 1.6. In the same disease Rosenow lays stress on the fact that the opsonic index is low-in fatal cases especially so. This analogy serves further to emphasise the intimate relationship of streptococci to scarlet fever. Four cases of nephritis were investigated, in the first and third of which strain $a$ was used, and in the second and fourth strains $d$ and $e$. As in the previous cases, the results obtained seem to be independent of the variety of streptococcus, the opsonin being the same for all the strains used; nor do they bear out French's suggestion, that the variation of opsonic content produced by injection of various strains of an organism may afford a means of distinguishing these.

CASE 9.-Moderately severe attack of scarlet fever; cervical adenitis and albuminuria appearing on seventeenth day of disease, with later anuria and vomiting. Blood in small quantity was present on the thirty-sixth day, and lasted twelve days; the albuminuria persisted for thirty days, during the latter part of the time only in traces.

Opsonic index on the 7 th day of nephritis (23rd day of disease), 0.5

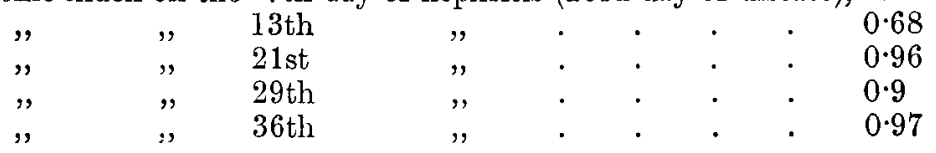

CASE 10.- Sharp attack of scarlet fever, the initial fever lasting seventeen days. Nephritis supervened on the nineteenth day-traces of blood and albumin being present for fifty days. The quantity of urine was always good, and general conditions excellent.

Opsonic index on the 11 th day of nephritis (30th day of disease), 0.87

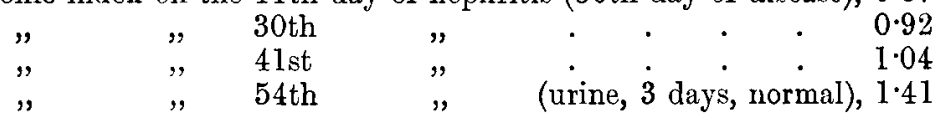


CASE 11.-With a fairly severe attack there was present a trace of albumin, which persisted in varying quantity for seventy days. Blood in considerable quantity was noted on the twenty-seventh day of the disease, and lasted intermittently for fifty days. There were thus periods of improvement, followed by increase in severity of the nephritis. Anæmia was a rather prominent feature in the later stages.

Opsonic index on the 2nd day of nephritis (28th day of disease), 0.71

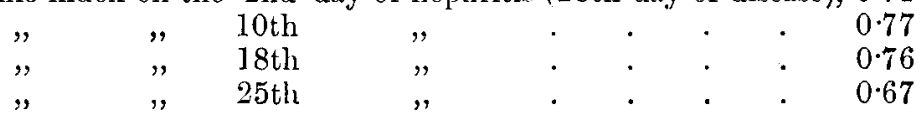

Unfortunately, I was unable to make further observations on this case. At the date of the last estimation there was just a trace of albumin present, and the condition seemed to be clearing up. The low index therefore came as a surprise, but has its explanation in the subsequent progress of the case-albumin persisting for nearly a month after, and much blood and renal débris being present on several occasions.

CASE 12.-Slight initial symptoms with normal convalescence till about a month after admission, when arthritis and much albumin and blood in the urine developed. Uræmic symptoms were marked on several occasions, and anæmia was profound. Blood was present for fifty days, and albumin even when discharged-four months after admission.

Opsonic index on the 2nd day of nephritis, 0.62

$\begin{array}{lllll}" & \text { 35th } & \text {. } & 0.57 \\ " & " & 56 \text { th } & , & 0.4 \\ " & \text { " } & 94 \text { th } & , & 0.86\end{array}$

The main features of these cases are-(1) the low opsonic content of the sera at the inception of the nephritis; (2) the maintained low index during the height of the disease; and (3) the subsequent rise to normal or approximately normal values as the condition improves. In Cases 9, 11, and 12 the first observation was made immediately after the onset of the complication, and in all a markedly low index is present; in Case 10 the first index refers to the eleventh day of disease, and is, as might be expected, somewhat higher. Again, in Case 12 , much the worst of the series, the index reaches a very low level $(0 \cdot 4)$, and this at a time when the child's condition was grave. In Case 9 recovery was rapid, and this is reflected in the opsonic curve. The deduction seems warranted that the more severe the nephritis the more likely is the opsonic index to remain low for a long period. Probably, also, lower absolute indices are present, though in view of Case 12 one must not attach very much prognostic significance to an exceptionally low index per se.

In a number of cases of nephritis I have made isolated estimations during the early stages of the disease, and in all a relatively low index was found, the average being 0.72 . As Wright and others have shown, in genito-urinary tuberculosis the opsonic index is almost always low, i.e. the lesion being a localised one, tuberculo-tropic substances are uniformly diminished in the serum of people so affected. 
The parallel evidently holds with reference to nephritis. Furthermore, the view that the streptococcus is the direct causative agent gains support from those observations, the pathological process being the direct result of organisms living in, and confined to, the kidneys. Streptococci have been demonstrated in the scarlatinal kidney with considerable frequency, either in situ or more commonly by cultural methods (Raskin, Guinon, and Bakes Gordon, 1898-1901, Baginsky, 1900 , Turner, 1895 , etc.). The presence of streptococci in the urine of scarlet fever patients is now well recognised. Courtois (1899) found in forty-two albuminous urines collected by catheter, streptococci thirty-two times (71 per cent.), and where no albumin was present, only in 27 per cent. of the urines examined. Considerable experimental work has been done on the elimination of bacteria (chiefly streptococci) via the kidneys by Tchistovitch (1900), Pavlovskje, and Métin (1900). The latter observes that when the tubes containing urine-got by catheter or section-show colonies of the microbe injected, there is always indication of a vascular or epithelial lesion, this statement harmonising with Courtois' findings. If, then, this view of the pathogenesis of scarlatinal nephritis be correct, the opsonic index may be a useful guide to appropriate treatment.

In two cases of cervical abscess occurring about the third week the following indices were obtained:-

CASE 13.-Defervescence occurred on fourth day of disease-a few days later slight fever and adenitis followed by abscess formation. The skin became extensively undermined, and the resulting wound was very slow in healing, the sinus discharging freely even when the child's general condition was all that could be desired.

Opsonic index 6 days after incision of abscess, 0.64

$\begin{array}{ccccc}" & 60 & , & & 0 \cdot 64 \\ " & 80 & , & , & 1.13 \text { (wound healed for } \\ & & & & \end{array}$

CASE 14.-Sharp attack of scarlet fever, with ulceration of tonsils and trace of albumin in the urine. Condition improved till the twenty-first day, when severe cervical adenitis occurred, which lasted about a fortnight. Albuminuria and irregular pyrexia persisted for about a month after the onset of the adenitis.

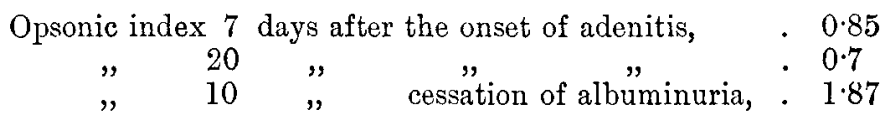

Here again low indices are noted while the inflammatory processes are going on, the opsonic power eventually rising in the latter case to the high figure of $1 \cdot 9$, and in the former to practically normal. The low index observed in Case 13 when the patient's general condition was very satisfactory, the wound, however, showing a striking disinclination to heal, illustrates a point not infrequently observed in tuberculous affections. 
There is a general agreement that no definite relationship exists between the leucocytes and the quantity of opsonin present in a given blood. In scarlet fever the leucocytic and opsonic curves are widely dissimilar : the former falls with the decline of temperature, and remains stationary at about the normal figure for the individual; the latter rises with the defervescence, and later exhibits characteristic fluctuations. Again, at the commencement of an attack of intercurrent nephritis or adenitis the leucocytic curve usually rises above normal, and may remain so for some time-even throughout the disease; the opsonic curve, on the contrary, is low for a longer or shorter period, and rises only as the condition improves.

\section{Conclusions.}

1. In cases of scarlet fever running a fairly normal course the opsonic power varies in a pretty definite and constant way. It is decreased during the early febrile period, and rises to normal or above normal with the defervescence and general decline of symptoms. It falls during the second and third weeks, and even in uncomplicated cases the opsonic index may be comparatively low. There is an increase to normal or over during the fourth and fifth weeks.

2. In fatal cases with severe angina, the opsonic power is markedly subnormal.

3. Complications alter the usual curve, causing both relative and absolute differences. Thus the opsonic power is decreased at the onset, and during the earlier period of albuminuria, severe nephritis and secondary adenitis; as convalescence is established the quantity of opsonin rises.

4. Support is lent to the view that nephritis is due mainly, if not entirely, to streptococcal invasion of the kidney.

5. The opsonic values do not furnish many data for prognosis, but in general a persistent low index during nephritis or other serious complication is an unfavourable sign.

6. A definite relationship to the Streptococcus scarlatince has been demonstrated, but there is no striking difference in the results obtained with typical and a-typical varieties respectively of this organism.

\section{REFERENCES.}

1. KLEIN . . . . . . . L.G.B. Rep., 1886-1887, xvi. 367.

2. Turner . . . . . . Guy's Hosp. Rep., London, 1895, Ser. 3, vol. li. p. 199.

3. Denys and Leclef . - La Cellule, Louvain, 1895, xi. 177.

4. Bordet . . . . . Ann. de l'Inst. Pasteur, Paris, 1895, tome ix. $462 ; 1896$, x. 104,$193 ; 1897$, xi. 177.

5. Denys and Marchand - Bull. Acad. roy. de méd. de Belg., Bruxelles, 1896, Ser. 4, vol. x. p. 229. 
6. Denys and Menves . . Bull. Acad. roy. de méd. de Belg., 1897, Ser. 4 , vol. xi. p. 403.

7. Mennes . . . . . Ztschr. f. Hyg., Leipzig, 1897, Bd. xxv. S. 413.

8. Marchand . . . . . Arch. de méd. expér. et d'anat. path., Paris, 1898 , tome x. p. 253.

9. Gondon . . . . . . L.G.B. Rep., 1900, xxix. p. $385 ; 1901$, xxx.

10. Courtors . . . . . . "Thèse de Paris," 1899.

11. Tchistovitci . . . . Ann. de l'Inst. Pasteur, Paris, 1900, tome xiv. p. 802.

12. Métin . . . . . . . Ibid., 1900, tome xiv. p. 415.

13. Baginsky and Somerfeld Berl. klin. Wchnschr., 1900, pp. 588, 618.

14. Wright and Windson . Journ. Hyg., 1902, vol. ii. p. 385.

15. Neufeld and Rimpau . . Deutsche Med. Wchnschr., Leipzig, 1904, Jahrg. xxx. S. 1458.

16. Ruediger. . . . . . Journ. Am. Med. Assoc., 1905, vol. xliv. p. 198.

17. Macdonald . . . . Trans. Path. Soc. London, 1906, vol. lvii. p. 45.

18. Hektoen . . . . . . Journ. Infect. Dis., 1906, vol. iii. p. 434.

19. Rosenow . . . . . . Ibid., 1906, vol. iii. p. 683 .

20. Tснiтch kine . . . . . Ann. de l'Inst. Pasteur, Paris, 1906, tome xx. p. 499.

*21. Raskin, Gunion, and Bakes, "Twentieth Cent. Pract. of Med.," vol. xiv. Quoted by FORCHHEIMER.

*22. PavlovskJe . . . . . Ztschrift. f. Hyg., 1900, Bd. xxxiii. S. 261. 